\title{
Los desafíos de las políticas y la gestión social en América Latina
}

Ernesto Cohen

\section{Introducción}

Las políticas sociales y su gestión se enfrentan hoy en América Latina a un desafío con múltiples aristas. Sin duda, los problemas que pretenden resolver tienen una magnitud tan gigantesca que genera desaliento. Esto es evidente cuando se analiza la situación social de la región.

La pobreza está aumentando en términos absolutos de manera sistemática desde 1990 y, desde comienzos del nuevo siglo, también lo está haciendo en valores relativos. Durante la década pasada 7 de cada 10 puestos de trabajo se generaron en el sector informal y paralelamente a ello, la histórica desigualdad en la distribución del ingreso se ha venido acentuando en la mayor parte de los países de América Latina, que así preserva el dudoso privilegio de ser la región que la riqueza peor distribuida en todo el mundo.

Ante este negro panorama hay solo una buena nueva. El gasto público social ha aumentado de manera sistemática desde comienzo de los 90 hasta la fecha. 
Dicho incremento fue del $58 \%$ entre el año 1990 y el 2000, y si bien, disminuyó posteriormente, el cambio fue sólo marginal.

¿Qué se ha hecho con esa enorme magnitud de recursos destinada a financiar las políticas sociales? Sin duda, hay restricciones de carácter estructural. La concentración del patrimonio y del ingreso, las enormes diferencias en la retribución, tamaño y densidad ocupacional en las familias, así como la lenta maduración de los efectos del aumento de la cobertura educativa, constituyen verdaderos parámetros en el combate a la pobreza.

Pero también, hay limitaciones que surgen de la forma en que las políticas sociales se diseñan e implementan. En primer lugar, la gestión social tradicional, asume que el impacto perseguido (cualesquiera sea) se producirá automáticamente como resultado de la mera entrega de los bienes o servicios a la población destinataria de los programas y proyectos en los que se traducen las políticas (basta con entregar raciones alimentarias a los más pobres para que su situación nutricional mejore... cosa que, obviamente, jamás ocurrió).

En segundo lugar, aunque no de menor importancia, aparece la masiva introducción de mecanismos de mercado en las políticas sociales, bajo el supuesto que permitirían mejorar su eficiencia e impacto. Desafortunadamente las lecciones que América Latina ha venido aprendiendo a través de la experiencia indican con toda claridad que el mercado en modo alguno, actúa de manera milagrosa. Tiene pre-requisitos básicos para su funcionamiento y al mismo tiempo exige su permanente regulación.

Para superar las limitaciones precedentes se propone abordar simultáneamente tres senderos:

- Superar el estilo tradicional de gestionar los programas y proyectos sociales haciendo del impacto el punto de partida obligado para su diseño e implementación.

- Aprender de la experiencia a través de la evaluación de impacto y el monitoreo, para que estos sean los instrumentos centrales de los que se base la gestión cotidiana y se viabilice la reprogramación.

- Generar un estilo más participativo en el proceso de diseño, gestión y evaluación de las políticas sociales para de esta manera aumentar la eficiencia en la utilización de los recursos y el impacto en los fines perseguidos.

Estamos absolutamente convencidos que la combinación de estos principios es esencial para hacer que las políticas sociales se ajusten de verdad a criterios de equidad que son su fundamento último.

\section{La situación social en América Latina}

\section{Evolución de la pobreza}

La superación de la pobreza se ha convertido desde hace dos décadas en un objetivo prioritario en la agenda de los organismos internacionales y de las grandes Conferencias de las Naciones Unidas. Así ha sucedido también en la Cumbre del Milenio (2000) $)^{1}$, cuyas metas en materia de progreso social constituyen un desafío asumido por los gobiernos. La primera y más importante propone reducir a la mitad, en 2015, la proporción de población en pobreza extrema existente en 1990.

La pobreza es un fenómeno multidimensional, lo que plantea dificultades para su definición operativa y para su medición. Aquí se la entenderá como la situación en que viven personas y hogares cuyos recursos económicos son insuficientes para satisfacer sus necesidades básicas.

Durante los años noventa, América Latina logró algún avance en la reducción 
de la proporción de población pobre e indigente, desde 48,3\% y 22,5\% en 1990 hasta $42,5 \%$ y $18,2 \%$, respectivamente, en 2001. Sin embargo, en los años posteriores, la situación ha empeorado: en 2003 el $43,9 \%$ de la población era pobre y 19,5\% de ella estaba en situación de indigencia (Gráfico 1). El número de pobres pasó de 200 millones en 1990 a 225 millones en 2003, mientras que los indigentes crecieron de 93 millones a 100 millones entre los mismos años (Gráfico 2).
En el ámbito de los países, la evolución de la pobreza y la indigencia entre 1999 y 2001/2002 también se caracterizó por variaciones más bien pequeñas, con pocas excepciones. Los únicos casos en que se produjo un marcado deterioro de las condiciones de vida de la población fueron Argentina y, en menor medida, Uruguay. En Argentina (área urbana), la tasa de pobreza casi se duplicó entre 1999 y 2002, al pasar de $23,7 \%$ a 45,4\%, mientras que la indigencia se multiplicó por tres, subiendo

Gráfico 1: Las nuevas cifras revelan un deterioro en la pobreza y la indigencia en América Latina en el período 2000-2002 ...

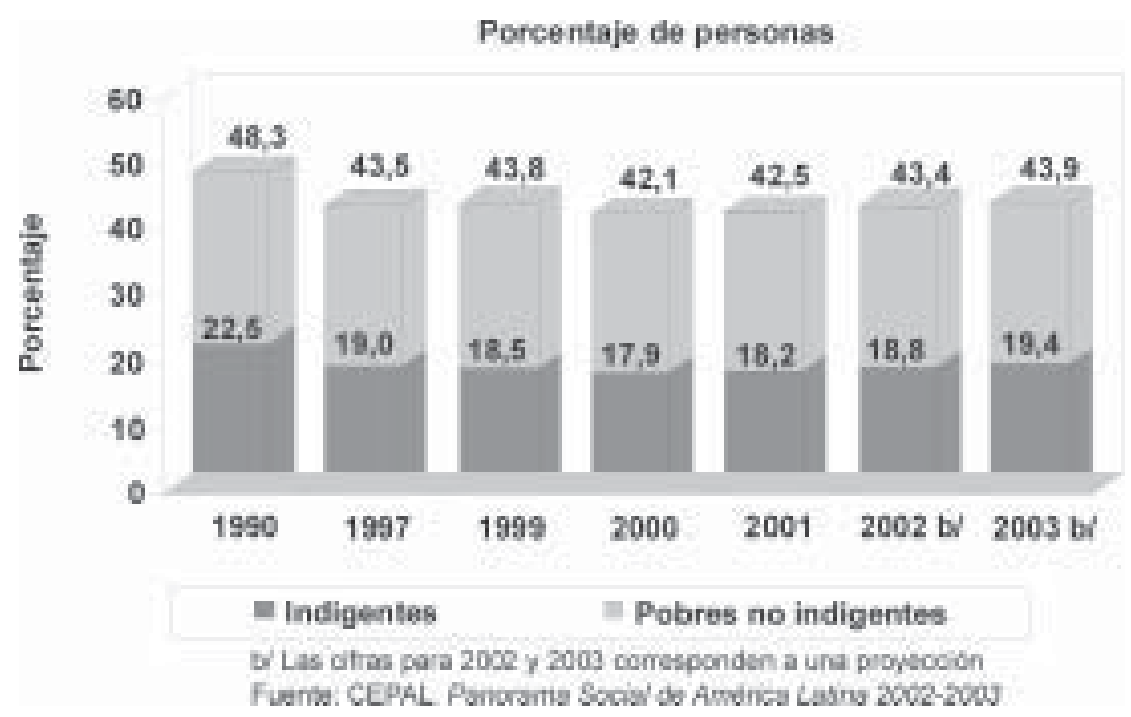

Gráfico 2: ... que se traduce en un mayor $\mathrm{n}^{\circ}$ de personas con niveles de vida insuficientes

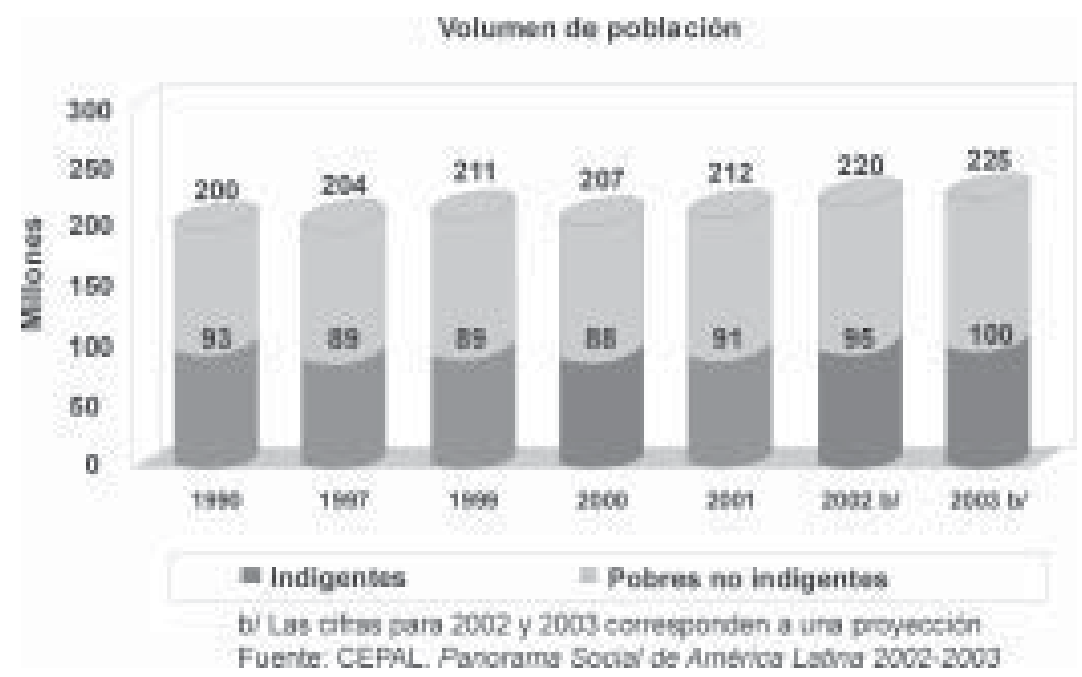


de 6,7\% a 20,9\%. Uruguay (área urbana) también registró un aumento significativo de la pobreza, que pasó de $9,4 \%$ a $15,4 \%$, aunque en este caso la indigencia mantuvo un nivel reducido, afectando tan solo al 2,5\% de la población.

La variedad de situaciones muestra que si bien las crisis globales afectan a todos los países, su impacto es desigual, entre otras cosas por el papel que juegan las políticas públicas que contribuyen a aliviar, aunque sea parcialmente, los choques externos y su impacto sobre los pobres.

\section{Empleo y desigualdad en la distribución del ingreso}

Entre 1990 y 1999 aproximadamente 40 millones de personas se incorporaron a la fuerza de trabajo en América Latina. De ellas casi 11 millones no encontraron trabajo o lo perdieron. De los 29 millones de ocupados casi 20 millones fueron al sector informal (Gráfico 4). Esto quiere decir que alrededor del 70\% de los nuevos empleos de la década se generaron fuera del sector formal de la economía.

Gráfico 3: La evolución de la pobreza entre 1997 y 2001-2002 también muestra distintas tendencias entre países

\section{Pobreza}

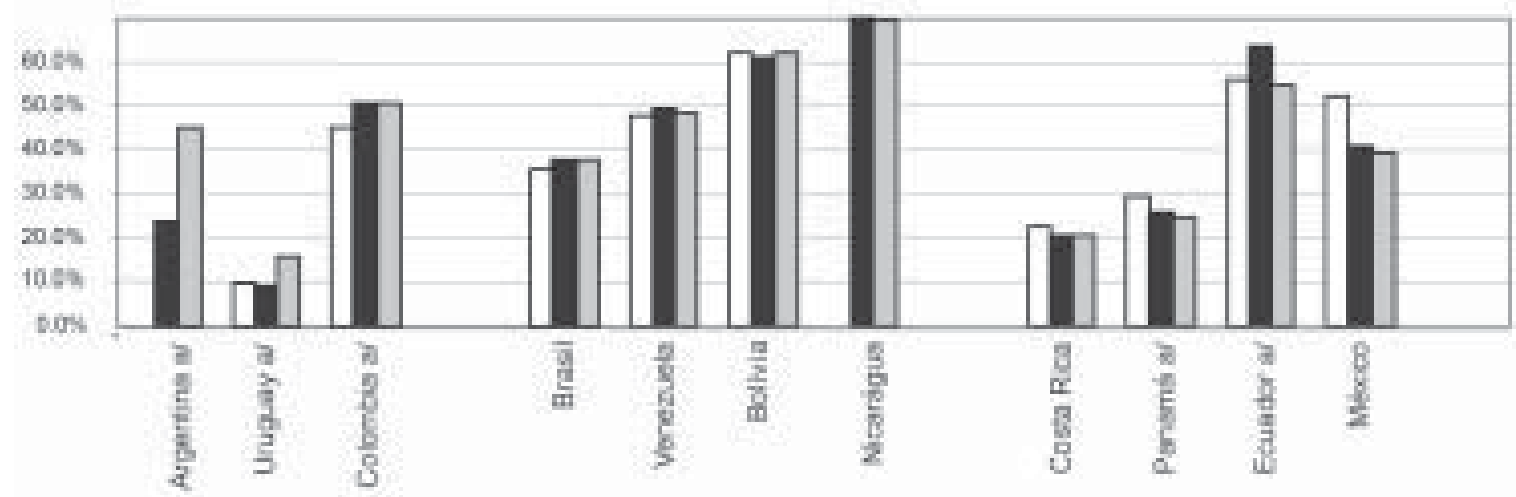

Indigencia

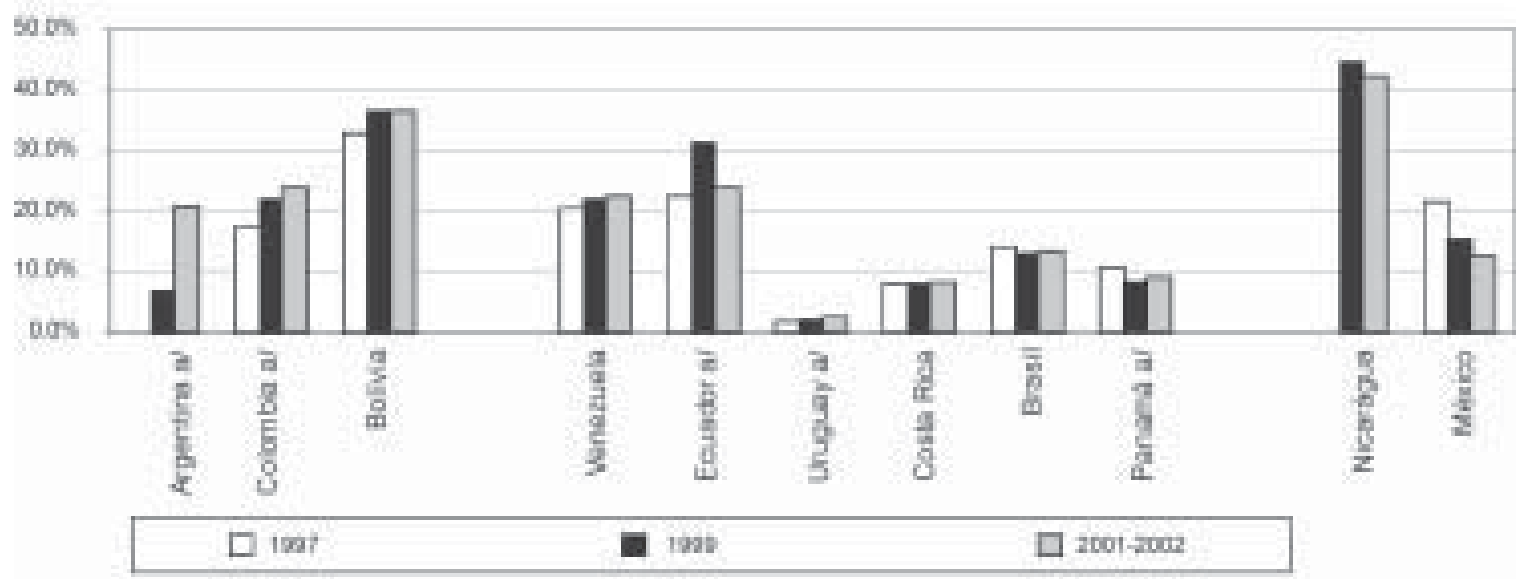

Di Areas urbanas

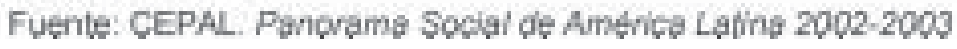


Esto tiene implicaciones importantes. El salario real de los informales en el año 2000 fue inferior al que tenían en 1980, al mismo tiempo que se incrementó de manera sistemática la brecha en las retribuciones de los formales e informales (los segundos tienen una remuneración media que es inferior a la mitad de los primeros).

El crecimiento económico es, sin duda, una condición necesaria para el abatimiento de la pobreza. Por ello es positivo, que luego de media década perdida (19972002), la región haya vuelto a crecer en 2003 $\mathrm{y}$ existan indicios que sugieren el inicio de una trayectoria ascendente ${ }^{1}$. Cabe suponer, entonces, que el producto por habitante, que se mantiene todavía por debajo del alcanzado en 1997 podría superar ese nivel.

Pero el volumen y persistencia de la pobreza encuentra, también explicación en la concentración del ingreso (Gráfico 5). Una menor desigualdad sería incluso favorable al crecimiento económico, porque "cuanto más desigual sea un país, menos efectivo será el crecimiento para reducir la pobreza" (Lustig et al., 2001). Se ha sostenido que, con la distribución actual no sería posible superar tasas de crecimiento de 3\% ó 4\% debido a que la mitad de la población no aporta el esfuerzo de crecimiento, dada su escasa participación en el ingreso nacional y a su pobreza (Birdsall, 1998). Todo ello conduciría a que hoy exista en la región una pobreza “innecesaria” (BERRY, 1997). De haberse mantenido la [ya concentrada] distribución del ingreso de comienzos de los años ochenta, el aumento del número de pobres derivado de la crisis de la deuda habría sido 50\% inferior (LONDOÑO; SZÉKELY, 1997). Por lo mismo, "Si América Latina tuviera la distribución del ingreso que corresponde a su nivel de desarrollo de acuerdo con los patrones internacionales, la incidencia de la pobreza sería la mitad de lo que es realmente" (BID, 1998).

La región se ha caracterizado secularmente por su mala distribución del ingreso.

Gráfico 4: La insuficiente generación de puestos de trabajo elevó el desempleo y la informalidad en las zonas urbanas

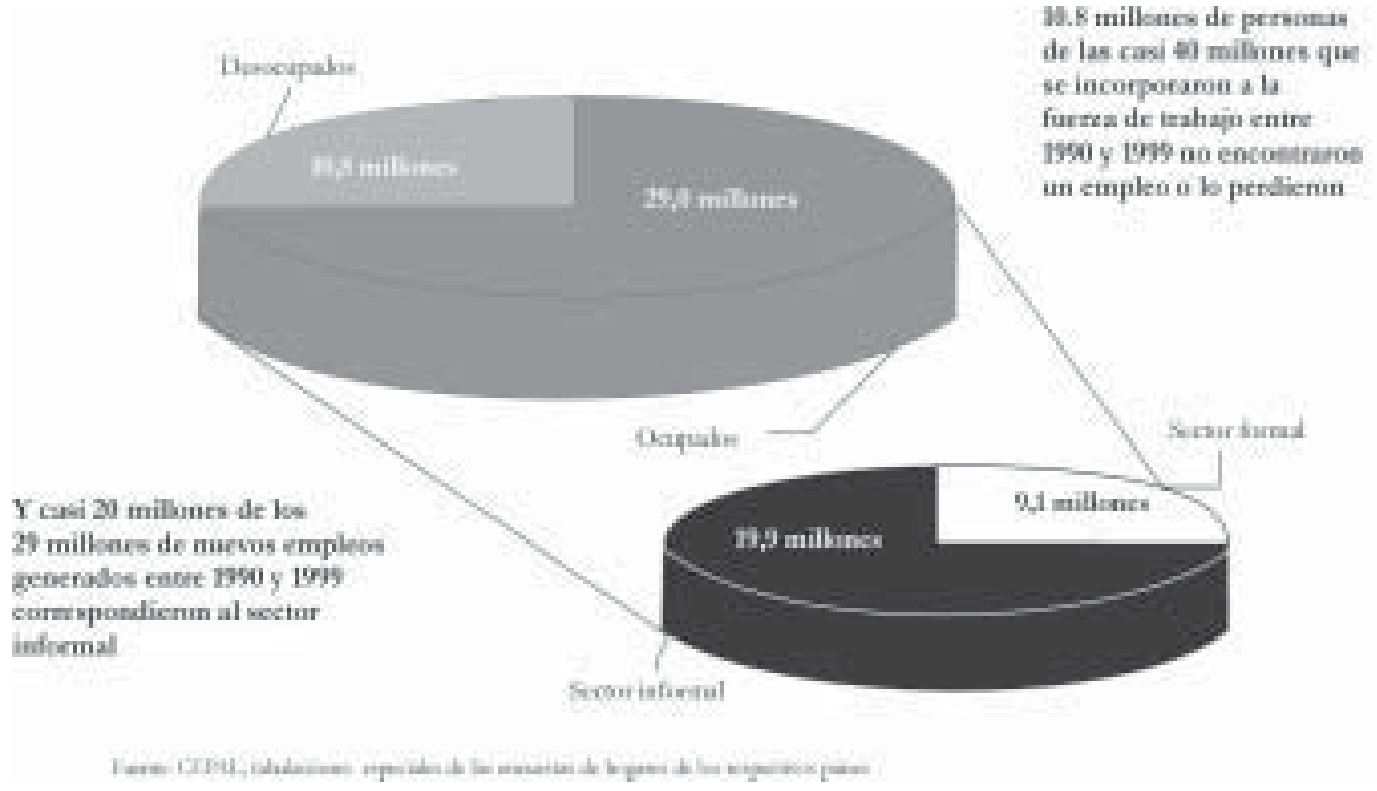


Incluso, durante la década de los noventa, ella empeoró en 7 países y sólo mejoró en 3 , para volver a deteriorarse en los primeros años de la actual década. Por consiguiente la distribución no mejora. Por el contrario, ha venido empeorando. Este proceso no cambiará como una consecuencia "natural" del crecimiento. Hay que promoverla a través de las políticas públicas, lo que no sólo exige voluntad política de llevarlas adelante, sino que también implica alterar los determinantes de la actual distribución del ingreso, que son de naturaleza patrimonial, demográfica, educacional, ocupacional y de remuneraciones, que resultan difícilmente modificables.

\section{Gasto público social}

El gasto público con el que se financian las políticas sociales creció más del 58\% durante la década pasada, pasando de US\$ 342 per cápita en 1990 a US\$ 540 en el año 2000. Las transferencias que surgen de la valoración monetaria de los bienes y servicios que entregan las políticas sociales representan un poco más del $43 \%$ de los ingresos del 20\% más pobre de América

Gráfico 5: América Latina (17 países): participación en el ingreso total del 40\% de los hogares más pobres y del 10\% más ricos, 1999 a/ (en porcentajes)

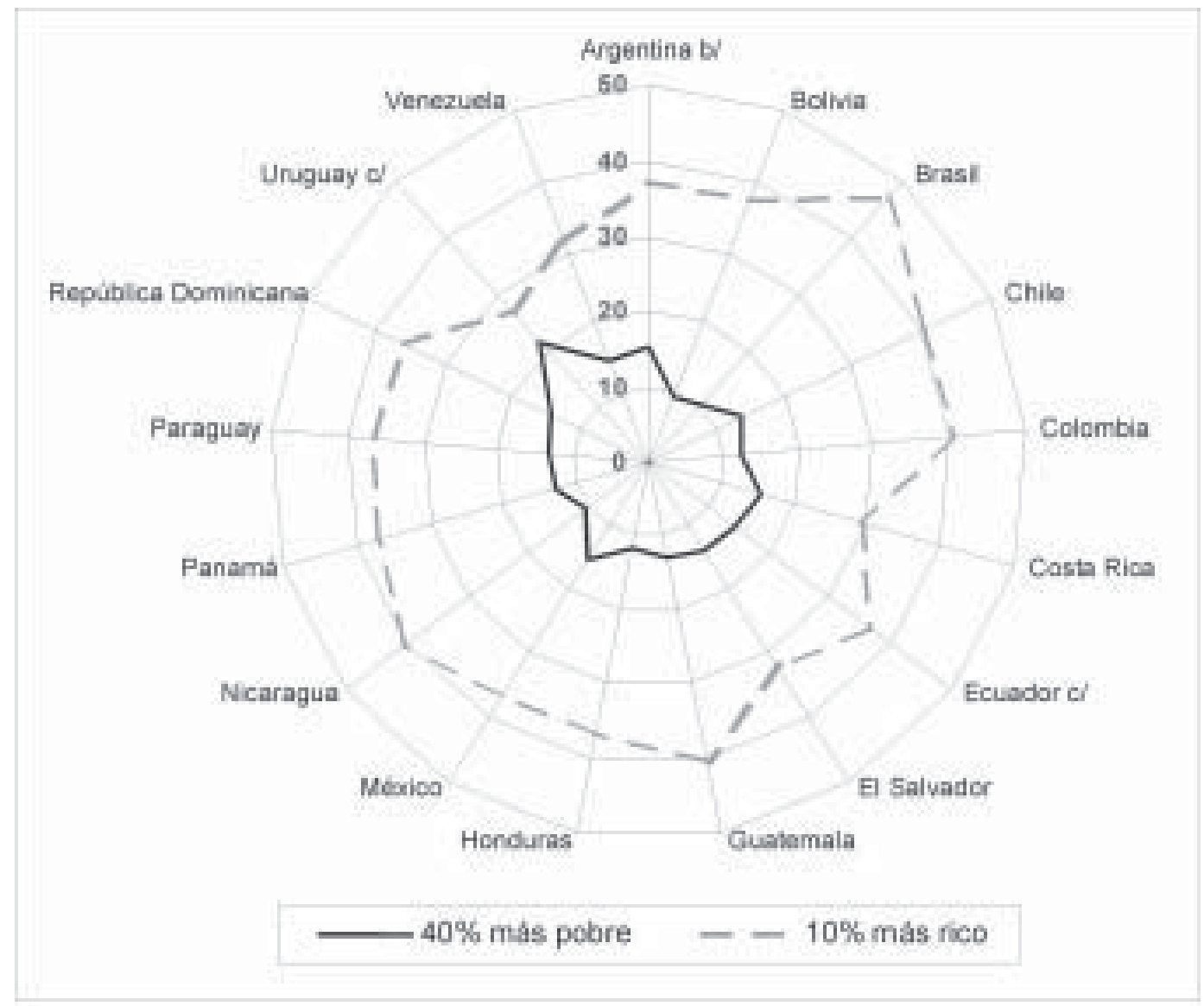

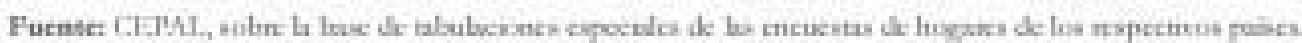

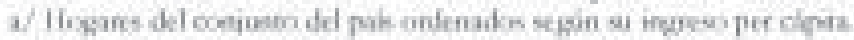

b. Gin Henre Ams

c) Total urtan. 
Latina. Por lo tanto, es evidente que el gasto público social es redistributivo, pero también se debe anotar, que no es lo suficientemente redistributivo: el 20\% más rico recibe un volumen mayor que el anteriormente mencionado y, en varios países, duplica lo destinado a los sectores más pobres. Sin duda que uno de los factores explicativos de lo dicho es el peso que tiene la seguridad social en el gasto público, que en algunos países se lleva más del $50 \%$ del total.

El nivel del gasto social es un mal indicador del desarrollo social porque no permite determinar si efectivamente se está alcanzando el impacto esperado en las poblaciones destinatarias (CEPAL, 1997, p. 187-188). Entre la asignación de recursos y el impacto causado existe una incógnita, porque no necesariamente los recursos gastados sirven a los fines para los que fueron destinados. El mero desempeño eficiente en la prestación y entrega de bienes o servicios no implica que se está contribuyendo a la solución de los problemas sociales.

La experiencia en la región muestra que con fuertes incrementos de gasto público social se pueden obtener resultados marginales, cuando la gestión de los programas es inadecuada. Para que exista impacto redistributivo del gasto social es necesario que exista mayor racionalidad en la asignación de recursos.

\section{Restricciones de la gestión social}

\section{El modelo tradicional de la gestión} social

El proceso de modernización del Estado tiende necesariamente a sustentarse en las estructuras institucionales existentes. Ello es una fuente de problemas en los

Gráfico 6: Fuerte aumento del gasto social por habitante en los noventa, aunque se mantuvo la heterogeneidad regional

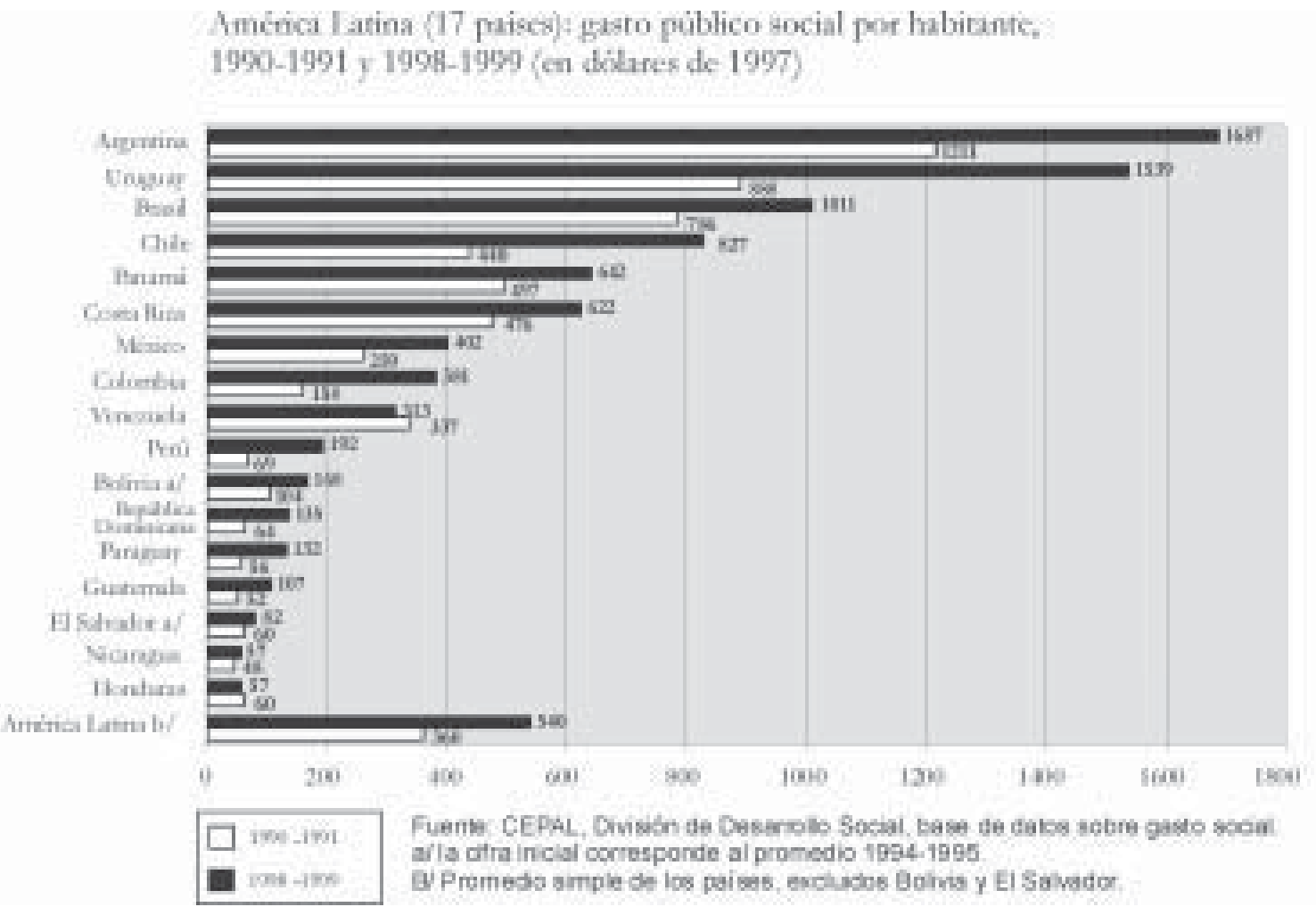


modelos de organización y gestión, que generan una lógica perversa en lo social.

Los modelos tradicionales de organización y de gestión de los programas sociales suelen asumir una relación determinística entre productos e impacto. Por ello, sus preocupaciones centrales son la los programas sociales; y utilizar como criterios para monitorear y evaluar el rendimiento medir a cuánta gente se sirve, con qué velocidad, qué porcentaje de solicitudes se llenan en un período de tiempo y cuánto cuesta cada unidad de producto.

Gráfico 7: América Latina (18 países): gasto social/PIB en 1990-1991, 1996-1997 y 2000-2001

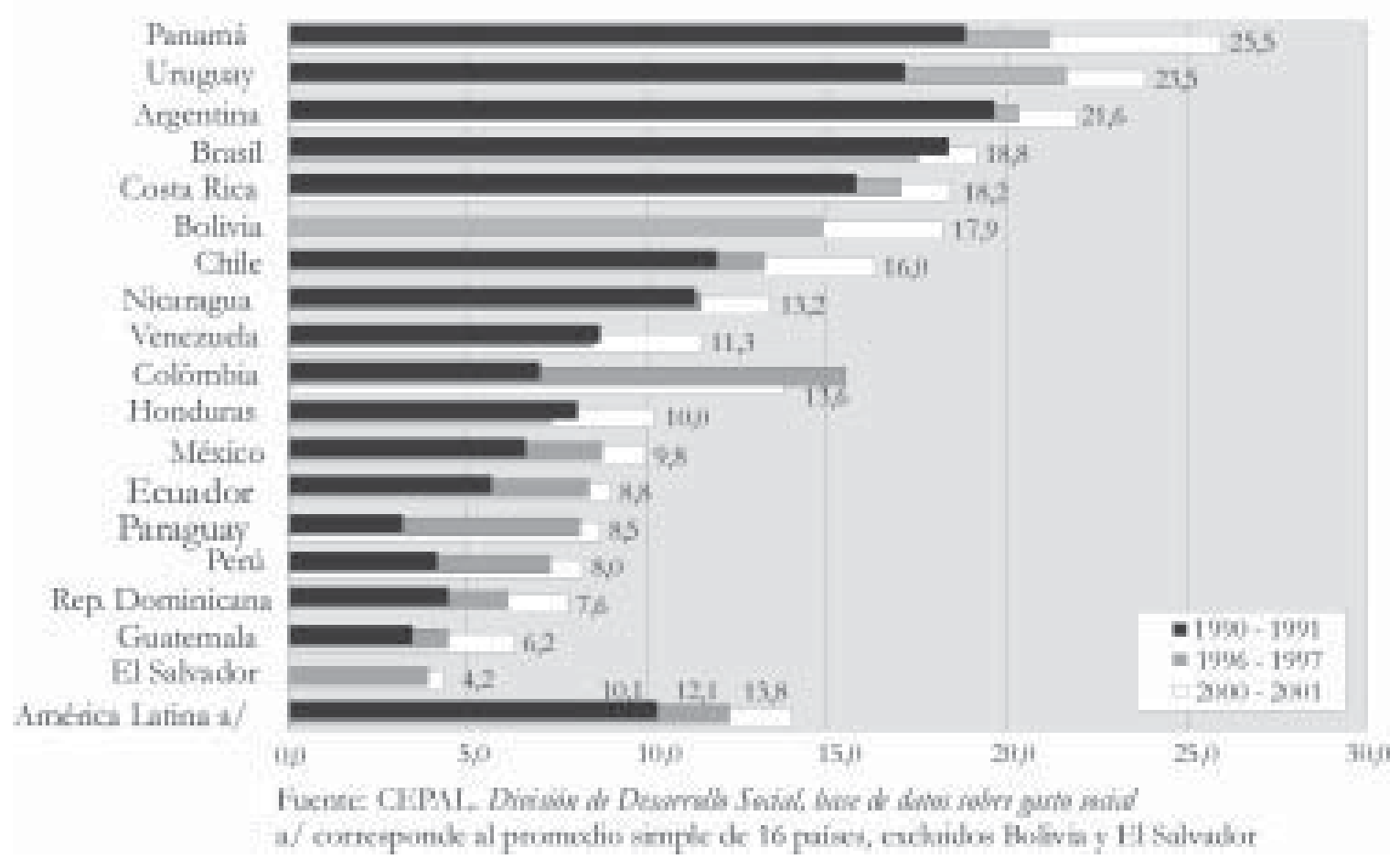

cobertura y los costos del programa y no prestan la atención que corresponde al impacto sobre sus destinatarios. Suponen que éste será satisfactorio en la medida en que se cumplan las metas de ejecución presupuestal, los objetivos de producción y la entrega de bienes y/o servicios a los niveles de cobertura prefijados. Esta concepción hace que el análisis de la gestión se centre en la eficiencia de la generación de los productos (bienes o servicios) y no en el impacto.

Lo anterior se plasma en dos pautas recurrentes: la adopción de un modelo monolítico de organización y gestión para
Esta estructura secuencial destaca que los programas y proyectos se insertan en un contexto institucional (generalmente un ministerio social) que tiene previamente definidos sus modelos de organización y gestión. En este marco se definen los procesos y actividades destinados a transformar los recursos en bienes o servicios (productos) que se entregarán a la población destinataria asumiendo que producirán el impacto buscado.

Pero, en la práctica, no sucede así. Las evaluaciones llevadas a cabo sobre programas sociales en América Latina muestran que tal supuesto no se puede sostener. 
El “esquema tradicional” tiene la siguiente lógica:

\section{Modelo de organización / Modelo de Gestión $\longrightarrow$ Processos/Actividades $\longrightarrow$ Productos}

\section{La introducción de mecanismos de mercado y sus limitaciones}

La introducción de mecanismos de mercado viene precedida por el otorgamiento al sector privado de un alto grado de racionalidad, acompañado de una confianza ilimitada en la presión que ejerce la competencia para motivar y regular el comportamiento de productores y consumidores.

Pero no debe olvidarse (Mendoza, 1991) que el mercado, no aparece por generación espontánea, de lo que da cuenta la penosa transición de los ex países socialistas. Nacen y se desarrollan (o desaparecen) como consecuencia de una interacción eficaz (o ineficaz) entre diferentes agentes y de un marco cultural que facilita (o dificulta) que las reglas del juego sean socialmente aceptadas.

Recientemente, la externalización ha irrumpido fuertemente en los programas sociales generando beneficios, pero también costos de transacción derivados de su inadecuada implementación, de la ausencia de marcos reguladores, o bien como producto del desconocimiento de la realidad institucional en los que se implementa.

Su utilización es muy amplia en los programas sociales. Más allá de la mera contratación de la producción y/o distribución de bienes o servicios, (complementación alimentaria, apoyo crediticio, capacitación o servicios de atención en salud), la contratación al exterior muchas veces incluye otras dimensiones de la gestión, tales como la selección de beneficiarios del programa, la evaluación ex-ante y ex-post de proyectos, así como la supervisión y la contratación de servicios especiales (desarrollo informático, estudios de costos, etc.). Las limitaciones que aparecen con mayor frecuencia en el proceso de externalización en América Latina son:

a) Debilidad en la creación de escenarios de mercado.

Para la externalización opere deben generarse escenarios que permitan operar las leyes de mercado. Frecuentemente los programas violan los requerimientos necesarios para introducir eficientemente este mecanismo. Las debilidades más frecuentes son:

- Oferta insuficiente de proveedores. En el diseño de los programas se asume la existencia de un mercado de oferentes (ONGs, empresas, etc.) suficientemente amplio y competitivo como para garantizar una calificada oferta de servicios. Este supuesto mercado suele ser, en realidad, muy pequeño o simplemente inexistente.

- La vulnerabilidad financiera es otra limitante de los proveedores. Una gran parte de ellos no cuenta con capital suficiente que les permita competir y subsistir de manera autónoma, volviéndose dependientes de la bolsa de recursos de los programas para los que trabajan. Por eso no logran generar estrategias de intervención integrales y de largo plazo, sino sólo intervenciones aisladas, que, en ocasiones, priorizan sus propios problemas por sobre los de la población objetivo. 
b) Fallas en la regulación de los mercados. Las condiciones de mercado necesarias para la externalización, no garantizan la obtención automática de beneficios. La gestión y regulación del mercado, son tanto o más importante que las condiciones para su operación. Las fallas más corrientes son:

- Déficit de regulación al externalizar la selección de beneficiarios. Entre las razones identificadas de selección adversa se encuentran la incapacidad técnica de los proveedores para realizar diagnósticos que conduzcan a una selección adecuada y las conductas oportunistas (cuando el proveedor actúa guiado por su propio interés en contra de lo contractualmente acordado).

Muchas veces se ha observado que un mismo ejecutar actúa como responsable tanto de la oferta de servicios como de la realización de diagnósticos y selección de beneficiarios, lo que fomenta el riesgo del oportunismo.

- Déficit en garantizar el acceso al servicio en todas las áreas geográficas que incluye el programa. Los proveedores guiados por el criterio de rentabilidad, limitan el acceso a beneficiarios a los que les cuesta más llegar, provocando situaciones de inequidad.

\section{Que hacer con las políticas y la gestión social}

\section{Superar el estilo tradicional de la gestión social}

Es preciso generar un enfoque más complejo de la gestión social, definida como la función de producción global, que

El "esquema innovador" busca transformar los insumos de la organización en productos, con arreglo a criterios de eficiencia interna e impacto externo sobre la población destinataria de los mismos. Este "esquema innovador" implica una inversión copernicana del anteriormente presentado.

Aquí, el punto de partida es el impacto. Éste determina la cantidad y calidad de los productos que se requieren para lograrlo. Exige una rigurosa justificación de la conexión causal existente entre productos e impactos. Así, es posible definir un programa de producción con los procesos y actividades requeridos para la transformación de los insumos en productos. Todo lo anterior plantea requerimientos específicos sobre los modelos de gestión y organización para que puedan responder adecuadamente a dicha cadena causal.

Dado que los programas sociales no se justifican por la utilización eficiente de los recursos que reciben sino por alcanzar los resultados que pretender obtener, se persigue maximizar el impacto y ello determina los modelos de gestión y organización.

\section{Aprender de la experiencia a tra-} vés de la evaluación de impacto y el monitoreo

Hasta ahora la evaluación de impacto es solo un fenómeno errático en el campo de las políticas sociales. Esto trae como consecuencia que los programas y proyectos derivados presentan interrogantes esenciales. En general, no se sabe si los programas sirven o no sirven; si sirven ¿cuánto sirven?

\section{Impacto $\longrightarrow$ Productos $\longrightarrow$ Procesos/Actividades $\longrightarrow$ Modelo de Gestión/Processos/Modelo de Administración}


$y$, quienes son los principales beneficiarios (o "perjudicatarios") de los mismos.

El monitoreo, por otro lado, siempre se menciona. En la práctica, no existe un solo programa o proyecto social que no declare tener un sistema de monitoreo. Pero otra es la verdad. Cuando los programas sociales se financian con préstamos de organismos multilaterales (como el Banco Mundial o el Banco Interamericano de Desarrollo) se realiza el monitoreo requerido para cubrir los requerimientos contables de las instituciones prestatarias, que se traduce operativamente en el seguimiento físico financiero de los acumulado en el campo de las políticas sociales permite, hoy, afirmar que los programas sociales con participación comunitaria en su diseño, gestión y evaluación, tienen resultados muy superiores a los tradicionales, burocráticos y verticales.

Como ejemplo de lo dicho, vale la pena mencionar un estudio que el Banco Mundial realizó en 1995 analizando 121 proyectos de dotación de agua potable para campesinos pobres en 49 países de Asia, África y América Latina. Los resultados obtenidos se presentan en el cuadro siguiente.

\section{Participación y rendimiento}

\begin{tabular}{|c|c|c|c|}
\hline \multirow{4}{*}{ Rendimiento } & \multicolumn{3}{|c|}{ Participación } \\
\cline { 2 - 4 } & & Alta & Baja \\
\cline { 2 - 4 } & Alto & $80 \%$ & $2,7 \%$ \\
\cline { 2 - 4 } & Medio & $20 \%$ & $40 \%$ \\
\cline { 2 - 4 } & Bajo & $0 \%$ & $57,3 \%$ \\
\hline
\end{tabular}

Fuente: Kligsberg (2000)

recursos asignados a las actividades programadas. Eventualmente, el papel de la Banca Multilateral es asumido por las instituciones nacionales (como el Ministerio de Hacienda o el de Economía), que establece el mismo tipo de requerimientos para realizar el seguimiento de los recursos que entregan para la implementación de las políticas públicas.

Lo dramático es que, en pocos o en ningún caso, el monitoreo se utiliza para el proceso de toma de decisiones en la gestión de los programas y proyectos que es la principal justificación de su existencia.

\section{Generar estilo más participativo} en los programas y proyectos

Es importante enfatizar que participar significa, en esencia, intervenir en el proceso de toma de decisiones. El conocimiento
Es evidente que los programas de proyectos sociales pueden ser llevados a cabo con y sin participación comunitaria. Pero, es hora de tomar conciencia prescindir de ella cobra altos precios en la eficiencia y el impacto de los mismos.

Que hacer con las políticas y la gestión social es una pregunta que no se contesta, llenando sólo los vacíos que presentan los capítulos antes mencionadas. Pero estamos totalmente convencidos que aplicar estos tres principios es esencial para hacer que las políticas sociales se ajusten de verdad a los criterios de equidad que son fundamento último. (Artigo recebido em outubro de 2005. Versão final em dezembro de 2005) 


\section{Notas}

${ }^{1}$ Empero, el entonces presidente del Banco Mundial James Wolfensohn denunció - en la Conferencia de Shangai, en mayo de 2004 - que el mundo se ha olvidado de luchar contra la pobreza: "Hoy en día se habla de boca para afuera sobre la reducción de la pobreza. Sin embargo, a la hora de la verdad, las palabras no se transforman en acciones". Asimismo, la Agenda de Shangai, sobre la reducción de la pobreza afirmó que las Metas del Milenio no se cumplirán en la mayoría de los países en desarrollo, particularmente en el Africa Subsahariana (EFE, 27 de mayo de 2004).

${ }^{2}$ Por ejemplo, la CEPAL espera que la región crezca alrededor de un 4\% en 2004. América del Sur crecerá 4,4\%; México y Centroamérica 3,4\% y el Caribe 2,9\%. Este mejor panorama deriva de la recuperación del crecimiento en las economías industrializadas. Los términos de intercambio serán favorables, en especial para los exportadores de petróleo y de minerales. Las mejores cifras serán Venezuela, $10,3 \%$, a partir del alza petrolera; de Uruguay, 7,5\%, impulsado por el sector exportador, tras cuatro años de recesión: de Argentina, 6,5\%, continuando su recuperación y Ecuador, 5,0\% (CEPAL, 2004).

\section{Referências bibliográficas}

Banco Interamericano de Desarrollo. Los objetivos dedesarrollo del Milenio en América Latina y el Caribe. Retos, accidentes y desafíos. Washington DC, enero, 2004. - América Latina frente a la desigualdad, Washington D.C.

Birdsall, N; David, R.; Sabor, R. La desigualdad como limitación para el crecimiento en América Latina, Oikos, n. 8, septiembre, 1995.

Cepal. La brecha de la equidad. América Latina, el Caribe y la Cumbre Social. Santiago (Chile), CEPAL, (LC/G.1954/Rev.1-P), 1997.

(Chile), 1998a. . Panorama Social de América Latina 1997, (LC/G.2050-P), Santiago

. Impacto de la crisis asiática en América Latina. Documento presentado al Período de Sesiones de la CEPAL, (LC/G.2026 - SES 27/23), Oranjestaad, Aruba, 1998b.

(Chile), 1999b.

. Panorama Social de América Latina 1998, (LC/G.2050-P), Santiago

. Panorama Social de América Latina 1999-2000, (LC/G.2068-P), Santiago (Chile), 2000a.

- La brecha de la equidad. Una segunda evaluación. Presentado a la Segunda Conferencia Regional de Seguimiento de la Cumbre Mundial sobre Desarrollo Social (LC/G.2096), Santiago (Chile), mayo, 2000 b. 
Panorama Social de América Latina 2000-2003. Santiago (Chile), 2003 a.

CePal/CElade/Bid. Impacto de las tendencias demográficas sobre los sectores sociales en América Latina, (LC/DEM/161). Santiago (Chile), 1996.

Cohen, E.; Franco, R. Evaluación de proyectos sociales. México DF: Siglo XXI Editores, 1992.

. Gestión social: ¿Cómo lograr eficiencia e impacto?. México: Siglo XXI Editores/CEPAL, 2004.

Franco, R.. Los paradigmas de la política social en América Latina. In: Revista de la CEPAL, n. 58, Santiago (Chile), 1996, pp. 9-22.

Franco, R. ; EspíndolA, E. La educación media, clave del crecimiento y la equidad, in Rama, G.W. (ed.) Alternativas de reforma de la educación secundaria. Washington DC: Banco Interamericano de Desarrollo, 2003.

Kligsberg, B. ¿Cómo reformar el Estado para enfrentar los desafíos sociales del 2000?. Instituciones y Desarrollo n. 6, Barcelona, 2000.

Lomniz, L. Cómo sobreviven los marginados. México D.F.: Siglo XXI Editores, 1974.

Londoño, J. L.; SzÉKely, M. Persistent poverty and excess inequality: Latin America, 1970-1995, IDB Working Paper Series IDB n. 357, Washington D.C., 1997

Lustig, N. et al. Reducción de la pobreza y crecimiento económico: la doble casualidad. Documento presentado al Seminario sobre Teoría del Desarrollo, CEPAL, Santiago (Chile), 2001.

Ocampo, J. A. Nuestra Agenda. Presentación del Secretario Ejecutivo en el Foro Conmemorativo del Quincuagésimo Aniversario de la CEPAL, Santiago de Chile, 26 de octubre de 1998. In: La CEPAL en sus 50 años. Notas de un seminario conmemorativo. Santiago (Chile), CEPAL (LC/G.2103.P), 2000. 


\section{Resumo - Resumen - Abstract}

\section{Os desafios das políticas e da gestão social na América Latina Ernesto Cohen}

A pobreza vem aumentando, em termos absolutos, de forma sistemática a partir de $1990 \mathrm{e}$, a partir do início do novo século, também em valores relativos. Há, entretanto, uma boa notícia: o gasto público social tem também aumentado de maneira sistemática desde então. O que tem sido feito com a enorme quantidade de recursos destinada a financiar as políticas sociais? Há, de um lado, limitações que emergem da forma em que elas (as políticas) são desenhadas e implementadas. A gestão social tradicional assume que o impacto perseguido se produzirá automaticamente como resultado da simples entrega de bens ou serviços à população destinatária. Verifica-se ainda a massiva introdução de mecanismos de mercado nas políticas sociais, pressupondo-se que isso permitiria melhorar sua eficiência e impacto. Levando em consideração tais limitações, sugerem-se neste artigo três caminhos, quais sejam: a superação do estilo tradicional de gerir os programas e projetos sociais; o aprendizado resultante do acúmulo de experiência em avaliação de impacto e em monitoramento; e a criação de um estilo mais participativo no processo de desenho, gestão e avaliação das políticas sociais.

Palavras-chave: pobreza; gestão social; avaliação e monitoramento

\section{Los desafíos de las políticas y la gestión social en América Latina Ernesto Cohen}

La pobreza está aumentando en términos absolutos de manera sistemática desde 1990 y, desde comienzos del nuevo siglo, también en valores relativos. Hay, sin embargo, una buena nueva: el gasto público social ha aumentado también de manera sistemática desde entonces. ¿Qué se ha hecho con la enorme magnitud de recursos destinada a financiar las políticas sociales? Hay, por un lado, limitaciones que surgen de la forma en que ellas se diseñan e implementan. La gestión social tradicional asume que el impacto perseguido se producirá automáticamente como resultado de la mera entrega de los bienes o servicios a la población destinataria. Aparece, además, la masiva introducción de mecanismos de mercado en las políticas sociales, bajo el supuesto que permitirín mejorar su eficiencia e impacto. Teniendo en cuenta las limitaciones precedentes, se sugieren en este artículo tres senderos, estos son: la superación del estilo tradicional de gestionar los programas y proyectos sociales; el aprendizaje por medio de la experiencia de la evaluación de impacto y del monitoreo; y la generación de un estilo más participativo en el proceso de diseño, gestión y evaluación de las políticas sociales.

Palabras clave: pobreza; gestión social; evaluación y monitoreo

\section{Social policies and management challenges in Latin America Ernesto Cohen}

Poverty has systematically been increasing in absolute terms since 1990, as well as in relative terms since the beginning of the new century. There is, however, good news: social expenditure has also been increasing systematically ever since. What has been done to the enormous magnitude of resources destined to finance social policies? There are, on the one hand, limitations that emerge from the way policies are designed and implemented. Traditional social management assumes that 
the pursued impact will be automatically produced as a result of the mere delivery of goods or services to the target population. One also observes the massive introduction of market mechanisms in social policies, presuming that it would lead to efficiency and impact improvements. Considering such limitations, this article suggests three courses of action, namely: overcoming the traditional style of managing social programs and projects; learning from the accumulated experience in monitoring and impact evaluation; and creating a more participative process for the design, management and evaluation of social policies.

Key words: poverty; social policy; evaluation and monitoring

Ernesto Cohen

Professor e pesquisador da Faculdade Latino-Americana de Ciências Sociais - FLACSO em Santiago (Chile). Especialista sênior da Divisão de Desenvolvimento Social da CEPAL. Doutor pela London School of Economics. 
